\title{
Do male and female beetles (Tenebrio molitor) respond differently to rat feces containing eggs from the tapeworm, Hymenolepis diminuta?
}

\author{
John F. Shea
}

Department of Biology, Gonzaga University, Spokane, USA; shea@gonzaga.edu

Received 13 March 2010; revised 19 April 2010; accepted 23 April 2010.

\begin{abstract}
Males and females often differ in their susceptibility and exposure to infection. Thus, they may also differ in their ability to avoid infection. The beetle, Tenebrio molitor, risks infection with cysticercoids when ingesting rat feces containing eggs of the tapeworm, Hymenolepis diminuta. Previous studies demonstrated that beetles sometimes prefer infective feces suggesting that the tapeworm influences the foraging behavior of beetles. After recording beetle movement in an arena containing infective and uninfective feces for one hour, sex main effects were not significant, but the effect of sex asked in trials was significant for males. Specifically, more male beetles occurred on the uninfective bait than on the infective bait. This observation suggests that male beetles avoid infective feces, which decreases their probability of infection with $\mathrm{H}$. diminuta. If the cost of infection is higher in males, then selection to avoid infective feces may act more strongly on males.
\end{abstract}

Keywords: Hymenolepis diminuta; Cysticercoid; Tenebrio molitor; Behavior; Coprophagy; Parasite Manipulation

\section{INTRODUCTION}

In general, male vertebrates tend to show a greater intensity and prevalence of parasites than females [1-3], while the same pattern is not observed as commonly in invertebrates [4]. However, there are some exceptions in which experimentally infected arthropods result in a male infection bias [5-7]. This male infection bias in invertebrates may be linked to differences in infection susceptibility that are immunological [8-9], differences in infection exposure that are behavioral [10-11], or some combination [12]. If susceptibility and exposure to infection differs by sex, then does avoidance of infection also differ by sex? The beetle-tapeworm system lends itself to the study of this question.

In the tapeworm Hymenolepis diminuta, adults live in the small intestines of rodents, the definitive host. Eggs pass out of the rodents in their feces. The cysticercoid, or larval stage, occurs in an insect (the intermediate host, most often a beetle) that becomes infected by feeding upon infective feces. The rodent completes the cycle when it ingests an infected beetle. Thus, the success of this parasite depends on rodent-to-beetle transmission, which depends upon consumption of the egg stage by the beetle.

Prior studies $[13,14]$ tested the fecal preference of groups of beetles, but did not examine unisex groups of beetles. One study [15] tested the fecal preference of tapeworm-infected male and female beetles (Tenebrio molitor) both in groups and as individuals. Only one study [5] examined unisex groups of uninfected beetles (T. molitor), which allows for sex comparisons while eliminating potentially confounding interactions between the sexes during the preference trial. The purpose of this study is to assess the relative importance of sex in fecal preference by recording beetle movement in an arena containing infective and uninfective feces at one-minute intervals for one hour.

\section{MATERIALS AND METHODS}

The "OSU Strain" [16] of H. diminuta was maintained in male Sprague-Dawley rats and beetles (T. molitor). Three rats were infected with 30 cysticercoids and maintained on commercial rodent chow and water. Three additional rats, obtained from the same commercial source, of identical age and from the same litter, were maintained under the same conditions as the infected rats to serve as the source of control (uninfective) feces. On the morning of the trials, rat cages were checked every 10 minutes, and fecal pellets were collected with forceps 
to minimize contamination by rat urine. After each trial, the infective fecal pellets were examined to verify the presence of $\mathrm{H}$. diminuta eggs.

Beetles were maintained on wheat bran, and small pieces of potato were added to the cultures on a regular basis. Pupae were removed from the cultures, and male and female pupae [17] were placed in separate dishes containing wheat bran. Beetles that emerged during a $24 \mathrm{~h}$ period were collected such that a daily cohort of beetles was maintained for both sexes.

Before the trial, seven to ten day-old male and female beetles (starved for two to three days) were placed in unisex groups of ten. To mitigate pseudo-replication, twelve groups of each sex were used in the preference trial in an alternating sequence. Each group was placed under a glass bowl in the center of a plastic arena under red light conditions. Beetles were provided with two types of bait: uninfective (control) or infective feces both of which had been collected within 10 minutes of defecation and randomly paired. Control feces were positioned randomly either in areas 1 and 3 or 2 and 4 (Figure 1) and infective feces were positioned in the opposing areas. A paper towel was used to mash the fecal pellets on the squares to prevent their displacement by beetles during the trial. Each bait area $\left(25 \mathrm{~mm}^{2}\right)$ was large enough for all beetles to occur simultaneously and was located in a quadrant containing no bait (defined as a non-bait area). After 15 minutes, the bowl was removed and the beetles' movements were videotaped for 60 minutes under red light conditions. During video play back, the number of beetles observed at each bait area (square) and non-bait area (quadrant) was recorded at one-minute intervals. These occurrences were then summed over the 60 minute trial to obtain the total number of beetle occurrences at infective and control baits as well as non-bait areas.

Preference was measured as the difference between the number of beetles occurring at infective and control baits at each minute of the 60 minute trial. The same calculation was performed for the number of beetles occurring in non-bait areas. These differences in the number of beetle occurrences in the bait and non-bait areas were analyzed with the Mixed procedure in SAS (v.8, Cary, NC) with sex and trial as class variables and sex nested in trial as a random effect. The response variable was defined as the number of beetles at control bait/non-bait minus the number of beetles at infected bait/non-bait. Thus, a difference of zero indicates no preference.

The maximum proportion of female and male beetles occurring at the bait areas for each 60-minute trial was plotted against the average proportion of beetles at the bait areas (after an arcsine square root transformation for both proportions), and the slopes were compared. The same was done for the maximum proportion of female and male beetles occurring in the non-bait areas.

\section{RESULTS}

The number of beetle occurrences did not differ between males and females in bait (Table 1; Mixed procedure, $P$ $=0.159)$ or non-bait areas $(P=0.873)$. However, more males occurred at the uninfective bait than at the infective bait $(X \pm \mathrm{SE}=1.58 \pm 0.54, P=0.008)$, while females did not differ in the number of occurrences at either bait $(X \pm \mathrm{SE}=0.46 \pm 0.54, P=0.412)$. The number of beetle occurrences in the non-bait areas did not differ between infected and control halves of the arena for males (Table 1; $X \pm \mathrm{SE}=0.30 \pm 0.28, P=0.292$ ) or for females $(X \pm \mathrm{SE}=0.24 \pm 0.28, P=0.403)$.

In a plot of the maximum number of beetles at each bait area against the average number of beetles at each bait area (data from Table 2), the slopes did not differ between males and females at the infective $\left(F_{1,20}=\right.$ $0.045, P=0.834)$ or the control bait areas $\left(F_{1,20}=2.75\right.$, $P=0.113)$. In a plot of the maximum number of beetles in each non-bait area against the average number of beetles in each non-bait area (data from Table 3), the slopes

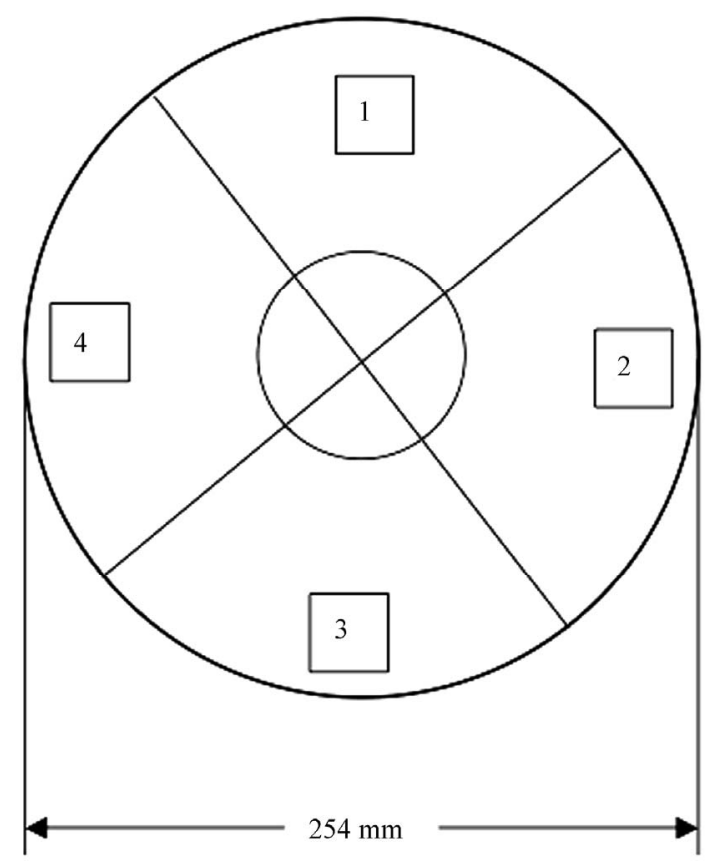

Figure 1. Diagram of the test arena for groups of beetles. Alternative baits were placed in the square bait areas located in four quadrants. Position of the control feces was determined randomly (e.g., either squares 1 and 3, or 2 and 4). Each bait area was $25 \mathrm{~mm}^{2}$ and located in a quadrant containing no bait defined as a non-bait area. The diameter of the center circle was $64 \mathrm{~mm}$ and the distance from its center to the edge of a square was $75 \mathrm{~mm}$. 
Table 1. Results of the mixed procedure (SAS v.8) for beetle preference by sex for the bait area (top), and for the non-bait (beetles in quadrant-beetles on bait) area (bottom) in group trials.

\begin{tabular}{lcccc}
\hline Effect & Mean difference estimate $^{\mathbf{1 , 2}}$ & s.e. & $\boldsymbol{t}$ & \multicolumn{1}{c}{$\boldsymbol{\boldsymbol { t }}$} \\
\hline Sex & -1.12 & 0.77 & -1.46 & 0.159 \\
Trial (males) & 1.58 & 0.54 & 2.90 & 0.008 \\
Trial (females) & 0.46 & 0.54 & 0.84 & 0.412 \\
& & & & \\
Sex & -0.06 & 0.40 & -0.16 & 0.873 \\
Trial (males) & 0.30 & 0.28 & 1.08 & 0.292 \\
Trial (females) & 0.24 & 0.28 & 0.85 & 0.403 \\
\hline
\end{tabular}

${ }^{1}$ For males and females, the mean difference equals total number of beetles at control bait/non-bait area minus total number of beetles at infected bait/non-bait area such that a positive value indicates preference for uninfective feces.

${ }^{2} n_{\bigcirc}=12, n_{+}=12$, d.f. $=22$ in all cases; data from Tables $\mathbf{2}$ and $\mathbf{3}$.

Table 2. Total number of beetles occurring at each bait type (infected or control) summed over the entire 60 minute trial (range in parenthesis) with mean number of beetles (standard errors in parenthesis).

\begin{tabular}{ccccc}
\hline & Males & & \multicolumn{2}{c}{ Females } \\
\hline Trial & Infected & Control & Infected & Control \\
1. & $34(0-3)$ & $299(0-8)$ & $26(0-4)$ & $22(0-1)$ \\
2. & $3(0-1)$ & $0(0-0)$ & $54(0-3)$ & $12(0-2)$ \\
3. & $36(0-3)$ & $243(0-7)$ & $22(0-3)$ & $45(0-3)$ \\
4. & $33(0-3)$ & $198(0-7)$ & $24(0-3)$ & $161(0-8)$ \\
5. & $73(0-7)$ & $173(0-7)$ & $36(0-2)$ & $71(0-5)$ \\
6. & $42(0-4)$ & $245(0-8)$ & $18(0-2)$ & $38(0-2)$ \\
7. & $192(0-7)$ & $34(0-2)$ & $9(0-1)$ & $90(0-5)$ \\
8. & $10(0-2)$ & $276(0-8)$ & $43(0-3)$ & $272(0-9)$ \\
9. & $34(0-3)$ & $1(0-1)$ & $202(0-6)$ & $71(0-4)$ \\
10. & $2(0-1)$ & $8(0-6)$ & $19(0-2)$ & $37(0-3)$ \\
11. & $141(0-5)$ & $182(0-6)$ & $20(0-2)$ & $36(0-2)$ \\
12. & $27(0-2)$ & $104(0-6)$ & $69(0-8)$ & $15(0-2)$ \\
Mean & $52.3(16.7)$ & $146.9(32.6)$ & $45.2(16.7)$ & $72.5(21.7)$ \\
\hline
\end{tabular}

Table 3. Total number of beetles occurring in the non-bait area for each half of the arena (infected or control) summed over the entire 60 minute trial (range in parenthesis) with mean number of beetles (standard errors in parenthesis).

\begin{tabular}{ccccc}
\hline & Males & & & Females \\
\hline Trial & Infected & Control & Infected & Control \\
1. & $150(0-8)$ & $117(0-5)$ & $254(0-10)$ & $298(0-9)$ \\
2. & $238(1-10)$ & $359(0-9)$ & $265(1-8)$ & $269(1-8)$ \\
3. & $179(0-7)$ & $142(0-6)$ & $244(0-7)$ & $289(2-9)$ \\
4. & $163(0-6)$ & $206(0-7)$ & $208(0-8)$ & $207(0-7)$ \\
5. & $164(0-8)$ & $190(0-7)$ & $215(0-8)$ & $278(1-8)$ \\
6. & $158(0-7)$ & $155(0-5)$ & $301(0-10)$ & $243(0-9)$ \\
7. & $208(0-8)$ & $166(0-9)$ & $204(0-7)$ & $297(2-9)$ \\
8. & $136(0-6)$ & $178(0-8)$ & $124(0-5)$ & $161(0-8)$ \\
9. & $206(1-6)$ & $359(4-8)$ & $160(0-7)$ & $167(0-6)$ \\
10. & $324(1-9)$ & $266(1-9)$ & $315(3-8)$ & $229(0-7)$ \\
11. & $153(0-7)$ & $126(0-6)$ & $265(0-9)$ & $279(0-10)$ \\
12. & $217(0-7)$ & $252(0-9)$ & $253(1-8)$ & $263(1-9)$ \\
Mean & $191.3(15.1)$ & $209.6(24)$ & $234(16)$ & $248.3(13.9)$ \\
\hline
\end{tabular}


did not differ between males and females at the infective $\left(F_{1,20}=0.211, P=0.651\right)$ or the control non-bait areas $\left(F_{1,20}=0.748, P=0.397\right)$.

\section{DISCUSSION}

The presence of parasites often restricts host movement $[18,19]$. In this study, male and female beetles did not differ in the number of occurrences in the non-bait regions suggesting that beetle movement is not restricted by the presence of feces or tapeworm eggs. Thus, fewer beetles at the infective feces suggest that male beetles avoid infective feces rather than feces in general. It is unlikely that the control bait repels males since the total number of males in the non-bait regions did not differ between infected and control halves of the arena. Also, male and females did not differ in the maximum number of occurrences at either the infective or control baits. This indicates that both sexes behave similarly when foraging in unisex groups, minimizing concern about the confounding influence of social interactions. Overall, these data indicate that male beetles avoid infective feces while females show no preference.

Two experiments with $T$. molitor examined the costs of infection on fertility. In the first, males mated singly with infected females produced an average $(n=30)$ of 115.2 ovulated eggs, while males mated with uninfected females produced an average $(n=30)$ of 127.9 ovulated eggs [20]. In the second, females mated singly with infected males produce an average $(n=7)$ of 37 larvae while females mated with uninfected males produce an average $(n=13)$ of 63.7 larvae [21]. Additionally, infected male T. molitor are less attractive to females [21] and exhibit a lowered response to pheromone produced by uninfected females [22]. Thus, infection in males not only results in less reproductive success, but infected males are less likely to mate with a female.

Further, experimental infections of T. molitor indicate that males are more susceptible to infection than females [5], although this may not be true in some strains of Tribolium confusum and Tribolium castaneum [23]. In addition, males are more exposed to infection when they consume more food [24]. If males are more susceptible and exposed to infection and incur a greater reproductive cost once infected, then selection to avoid infective feces may act stronger on males than on females. Females also incur a cost when infected [20] and so may also avoid infective feces, but this behavior was not detected in this experiment.

Beetle age may factor into the relative cost of infection. The finding that starved male beetles avoid infective feces only partially agrees with Pappas et al. who found that fed male beetles avoid infective feces while more fed and starved females fed upon infective feces [5]. Pappas et al. tested 14-18 day old beetles whereas this study tested seven to ten day old beetles. Repeating this experiment with recently emerged beetles could yield different results. This is because greater reproductive output occurs when female beetles are mated with males infected two days post-emergence, suggesting an example of fecundity compensation [25]. Further both males and females experience increased survivorship when infected two days post-emergence [26]. Thus, recently emerged beetles may experience less selective pressure to avoid infective feces.

Although the results suggest that males avoid infective feces, the effectiveness of this avoidance behavior in natural settings needs examination because one study of natural populations of Tenebrio molitor found that 51\% of males were infected as opposed to $41 \%$ of infected females [27]. Also, beetles produce both sex and aggregation pheromones $[28,29]$, which could influence individual foraging behavior, suggesting a need for experiments that track marked individual beetles.

\section{AKNOWLEDGEMENTS}

This study reflects work done while at The Ohio State University. I thank Jerry Downhower, Peter Pappas, Tom Waite and Larry Phelan for their helpful comments in the preparation of this manuscript. I especially thank Peter Pappas for the use of his laboratory equipment. Finally, I thank Yimei He for her statistical advice.

\section{REFERENCES}

[1] Zuk, M. and McKean, K.A. (1996) Sex differences in parasitic infections: Patterns and processes. International Journal for Parasitology, 26(10), 1009-1024.

[2] Poulin, R. (1996) Sexual inequalities in helminth infections: A cost of being a male? The American Naturalist, 147(2), 287-295.

[3] Schalk, G. and Forbes, M.R. (1997) Male biases in parasitism of mammals: Effects of study type, host, age, and parasite taxon. Oikos, 78(1), 67-74.

[4] Sheridan, L.A., Poulin, R., Ward, D.F. and Zuk, M. (2000) Sex differences in parasitic infections among arthropod hosts: Is there is a male bias? Oikos, 88(2), 327-334.

[5] Pappas, P.W., Marschall, E.A., Morrison, S.E., Durka, G.M. and Daniel, C.S. (1995) Increased coprophagic activity of the beetle, tenebrio molitor, on feces containing eggs of the tapeworm, hymenolepis diminuta. International Journal for Parasitology, 25(10), 1179-1184.

[6] Gray, D.A. (1998) Sex differences in susceptibility of house crickets, acheta domesticus, to experimental infection with serratia liquefaciens. Journal of Invertebrate Pathology, 71(3), 288-289.

[7] Wedekind, C. and Jakobsen, P.J. (1998) Male-biased susceptibility to helminth infection: An experimental test with a copepod. Oikos, 81(3), 458-462. 
[8] Kurtz, J., Wiesner, A., Götz, P. and Sauer, K.P. (2000) Gender differences and individual variation in the immune system of the scorpionfly panorpa vulgaris (Insecta: Mecoptera). Developmental and Comparative Immunology, 24(1), 1-12.

[9] Adamo, S.A., Jensen, M. and Younger, M. (2001) Changes in lifetime immunocompetence in male and female gryllus texensis (formerly G. integer): Trade-offs between immunity and reproduction. Animal Behaviour, 62(3), 417-425.

[10] Reimchen, T.E. and Nosil, P. (2001) Ecological causes of sex-biased parasitism in threespine stickleback. Biological Journal of the Linnean Society, 73(1), 51-63.

[11] Hecker, K.R., Forbes, M.R. and Leonard, N.J. (2002) Parasitism of damselflies (Enallagma boreale) by gregarines: sex biases and relations to adult survivorship. Canadian Journal of Zoology, 80(1), 162-168.

[12] Klein, S.L. (2000) The effects of hormones on sex differences in infection: From genes to behavior. Neuroscience and Biobehavioral Reviews, 24(6), 627-638.

[13] Evans, W.S., Hardy, M.C., Singh, R., Moodie, G.E. and Cote, J.J. (1992) Effect of the rat tapeworm, hymenolepis diminuta, on the coprophagic activity of its intermediate host, tribolium confusum. Canadian Journal of Zoology, 70(12), 2311-2314.

[14] Shostak, A.W. and Smyth, K.A. (1998) Activity of flour beetles (Tribolium confusum) in the presence of feces from rats infected with rat tapeworm (Hymenolepis diminuta). Canadian Journal of Zoology, 76(8), 14721479.

[15] Shea, J.F. (2007) Lack of preference for infective faeces in Hymenolepis diminuta-infected beetles (Tenebrio molitor). Journal of Helminthology, 81(3), 293-299.

[16] Pappas, P.W. and Leiby, D.A. (1986) Variation in the sizes of eggs and oncospheres and the numbers and distributions of testes in the tapeworm, hymenolepis diminuta. Journal of Parasitology, 72(3), 383-391.

[17] Bhattacharya, A.K., Ameel, J.J. and Waldebauer, G.P. (1970) A method for sexing living pupal and adult yellow mealworms. Annals of the Entomological Society of America, 63, 1783-1785.

[18] Van der Wal, R., Irvine, J., Stien, A., Shepherd, N. and Albon, S.D. (2000) Faecal avoidance and the risk of infection by nematodes in a natural population of reindeer. Oecologica, 124(1), 19-25.
[19] Hutchings, M.R., Kryriazakis, I., Anderson, D.H., Gordon, I.J. and Coop, R.L. (1998) Behavioural strategies used by parasitized and non-parasitized sheep to avoid ingestion of gastro-intestinal nematodes associated with faeces. Animal Science, 67, 97-106.

[20] Hurd, H. and Arme, C. (1986) Hymenolepis diminuta: The effects of metacestodes upon egg production and viability in the intermediate host tenebrio molitor. Journal of Invertebrate Pathology, 47(2), 225-230.

[21] Worden, B.D., Parker, P.G. and Pappas, P.W. (2000) Parasites reduce the attractiveness and reproductive success in male grain beetles. Animal Behaviour, 59(3), 543-550.

[22] Hurd, H. and Parry, G. (1991) Metacestode-induced depression of the production of, and response to, sex pheromone in the intermediate host tenebrio molitor. Journal of Invertebrate Pathology, 58(1), 82-87.

[23] Yan, G. and Norman, S. (1995) Infection of tribolium beetles with a tapeworm: Variation in susceptibility within and between beetle species and among genetic strains. The Journal of Parasitology, 81(1), 37-42.

[24] Shea, J.F. (2005) The effect of Hymenolepis diminuta (Cestoda) cysticercoids on the weight change, frass production, and food intake of the intermediate host, tenebrio molitor (Coleoptera). Parasitology Research, 98(1), 1-4.

[25] Hurd, H. and Ardin, R. (2003) Infection increases the value of nuptial gifts, and hence male reproductive success, in the hymenolepis diminuta-tenebrio molitor association. Proceedings of the Royal Society of London B, 270, S172-S174.

[26] Hurd, H., Warr, E. and Polwart, A. (2001) A parasite that increases host lifespan. Proceedings of the Royal Society of London B, 268(1477), 1749-1753.

[27] Rau, M.E. (1979) The frequency distribution of Hymenolepis diminuta cysticercoids in natural, sympatric populations of Tenebrio molitor and T. obscurus. International Journal for Parasitology, 9(2), 85-87.

[28] Tschinkel, W., Willson, C. and Bern, H. (1967) Sex pheromone of the mealworm beetle. (Tenebrio molitor). Journal of Experimental Zoology, 164(1), 81-85.

[29] Hurd, H. and Fogo, S. (1991) Changes induced by Hymenolepis diminuta (Cestoda) in the behaviour of the intermediate host Tenebrio molitor (Coleoptera). Canadian Journal of Zoology, 69(9), 2291-2294. 